\title{
The 3D Structure of the Galactic Bulge
}

\author{
Manuela Zoccali ${ }^{1,2,4}$ and Elena Valenti ${ }^{3}$ \\ ${ }^{1}$ Instituto de Astrofísica, Pontificia Universidad Católica de Chile, Av. Vicuña Mackenna 4860, 782-0436 Macul, Santiago, Chile \\ ${ }^{2}$ Millennium Institute of Astrophysics, Av. Vicuña Mackenna 4860, 782-0436 Macul, Santiago, Chile \\ ${ }^{3}$ European Southern Observatory, Karl-Schwarzschild Strasse 2, D-85748 Garching, Germany \\ ${ }^{4}$ Email: mzoccali@astro.puc.cl
}

(ReCEIVEd November 14, 2015; ACCEPTED December 21, 2015)

\begin{abstract}
We review the observational evidences concerning the three-dimensional structure of the Galactic bulge. Although the inner few kpc of our Galaxy are normally referred to as the bulge, all the observations demonstrate that this region is dominated by a bar, i.e., the bulge is a bar. The bar has a boxy/peanut (X-shaped) structure in its outer regions, while it seems to become less and less elongated in its innermost region. A thinner and longer structure departing from the main bar has also been found, although the observational evidences that support the scenario of two separate structures has been recently challenged. Metal-poor stars $([\mathrm{Fe} / \mathrm{H}] \lesssim-0.5 \mathrm{dex})$ trace a different structure, and also have different kinematics.
\end{abstract}

Keywords: galaxy: bulge - galaxy: structure - galaxy: stellar content

\section{INTRODUCTION}

In the last few years, Galactic archaeology has risen as a branch of astrophysics that seeks to unveil the origin of a stellar system by studying fossil records in its oldest stars. The oldest stars of the Milky Way have been found in the halo. It is in the halo where we can find traces of the Galaxy's primordial components, including streams of accreted satellites. The halo, however, makes up only about $1 \%$ of the total stellar mass of the Milky Way $\left(<10^{9} \mathrm{M}_{\odot}\right.$; Robin et al. 2003; Bell et al. 2008). If we seek to understand how the bulk of the Milky Way formed, a valuable alternative is to study the best compromise between old and massive, that is the Galactic bulge.

As reviewed elsewhere in this volume (e.g., paper by Gerhard), the mass of the Galactic bulge is rather poorly constrained. Most determinations cluster close to $1.5 \times 10^{10}$ $\mathrm{M}_{\odot}$, although a few authors find values as large as $2 \times 10^{10}$ $\mathbf{M}_{\odot}$ (Valenti et al. 2015) or as small as $6 \times 10^{9} \mathrm{M}_{\odot}$ (Robin et al. 2012). Even with this rather large scatter, the mass of the bulge must be close to $1 / 5$ of the total stellar mass of the Milky Way, and about ten times larger than the mass of the halo. The bulge age distribution is also currently debated, but all the studies agree that the bulk of bulge stars is $\sim 10$ Gyr old (Ortolani et al. 1995; Kuijken \& Rich 2002; Zoccali et al. 2003; Sahu et al. 2006; Clarkson et al. 2011; Valenti et al. 2013), with discrepancies regard- ing only the presence, and the number fraction, of an intermediate age tail of the distribution (see e.g. Bensby et al. 2011, 2013).

In addition to being old and massive, the Galactic bulge is the only galaxy bulge that can be resolved down to its faintest stars: a unique case that can be studied with exquisite details.

Kormendy \& Kennicutt (2004) classified galaxy bulges as classical bulges and pseudo-bulges. Classical bulges would be spheroids formed by gravitational collapse, or hierarchical merging of smaller galaxies. They would be formed in the very early epoch of galaxy formation, and thus are usually older than the disk. Pseudo-bulges, on the contrary, would be smaller, disk-like structures found in the innermost regions of spirals, and originated because the presence of bars favours the accumulations of gas in the very inner part of the disks, forming either an inner disk or a ring. Once the disk/ring is sufficiently massive, it starts forming stars that can be detected as young population in the central part of the disks. This classification has been used widely, often with the over simplification that spheroids would be classical bulges, while bars would be pseudo-bulges, because they are originated from disk instabilities. Through this paper, we will abandon this classification, mostly because recent observations of high-redshift galaxies suggest that the origin of bulges can be much more complex, likely related to the merging of dense, star-forming clumps present in the disk (Immeli et al. 2004; Carollo et al. 2007; Elmegreen, 
Bournaud, \& Elmegreen 2008; Bournaud, Elmegreen, \& Martig 2009). Hence, in what follows we will refer to the 'bulge' as the Milky Way region within a radius of $\sim 2-3$ kpc from the Galactic centre, without any implication on its nature or origin.

This paper reviews the progress of our knowledge of the bulge three-dimensional (3D) structure, focussing on the observational results. We occasionally present an updated version of the relevant figures, made using the state of the art observational data. Section 2 presents evidences for the presence of a main bar in the central region of the Milky Way. Section 3 explains how we became aware of a boxy/peanut (i.e., X-shaped) structure in the outer bulge. In Section 3, we discuss the $3 \mathrm{D}$ traced by the metal-poor stars in the bulge, with special emphasis on the spatial distribution of RR Lyrae (RRL) variables. Section 4 presents evidences for the presence of a distinct structure within a radius of $\sim 250 \mathrm{pc}$, and finally Section 5 reviews evidences for the presence of a longer and thinner bar, in addition to the main one.

\section{THE MAIN GALACTIC BAR}

\subsection{Early evidences}

The presence of a bar in the inner Milky Way was first suggested by de Vaucouleurs (1964) as a way to explain the departures from circular motions seen in the HI line profile at 21 $\mathrm{cm}$, for longitudes close to the Galactic centre. Nonetheless, the first direct evidence of the presence of a bar was presented by Blitz \& Spergel (1991) who analysed the $2.4 \mu \mathrm{m}$ maps of the Galactic centre by Matsumoto et al. (1982). Curiously, these authors claimed that the Galactic bar was associated with the peanut-shaped structure seen in the COBE maps by Hauser et al. (1990), while a separate and more metal-poor triaxial spheroid was responsible of the non-circular motions in the HI line-of-sight velocity versus longitude maps. Much larger than the bar, their triaxial spheroid extended up to the solar radius.

After correcting the COBE DIRBE maps by extinction, it was soon clear that the apparent peanut shape was only due to a dark cloud, known as the Pipe Nebula, close to $(l, b)=(0,5)$ (Weiland et al. 1994). The boxy structure, instead, was confirmed and interpreted as the signature of an edge-on bar, with the near side in the first quadrant, and the major axis at an angle $\theta=20^{\circ} \pm 10^{\circ}$ with the SunGalactic centre direction (Dwek et al. 1995). Indeed, the boxy isophotes of the near-IR COBE maps show an asymmetry along the longitude direction, with the positive-longitude half being brighter than the negative-longitude one. Both the pivot angle and the axis ratio $(1: 0.3: 0.2)$ measured by Dwek et al. (1995) are in excellent agreement with the most recent measurements based on resolved stars (see below). The same authors assume that the whole bulge is bar-shaped, and derive the first photometric mass of the bulge/bar. From the total $L_{K}$ luminosity and the fuel consumption theory, they estimate the progenitor mass of the post main-sequence stars, and then integrate a Salpeter IMF along the main sequence down to $0.1 \mathrm{M}_{\odot}$, obtaining $\mathrm{M}_{\mathrm{BULGE}}=1.3 \times 10^{10} \mathrm{M}_{\odot}$. The total $K$ luminosity measured by Dwek et al. (1995), $4.1 \times 10^{8} \mathrm{~L}_{\odot}$, is more than one order of magnitude lower than the value $\left(1.2 \times 10^{10} \mathrm{~L}_{\odot}\right)$ measured by Kent, Dame, \& Fazio (1991), using maps from the SPACELAB infrared telescope. By accident, however, the bulge photometric mass quoted above is remarkably similar to the dynamical mass derived by Kent $\left(1992,1.2 \times 10^{10} \mathrm{M}_{\odot}\right)$ because Dwek et al. (1995) assume a Salpeter IMF down to $0.1 \mathrm{M}_{\odot}$, significantly steeper than the observed one, as measured with near IR and optical star counts by Zoccali et al. (2000) and Calamida et al. (2015), respectively.

By using a different and 3D extinction correction on the same COBE/DIRBE data, Binney, Gerhard, \& Spergel (1997) also constructed a photometric model of the inner Milky Way, confirming a pivot angle $\theta \approx 20^{\circ}$, for a bar with approximate axis ratios (1:0.6:0.4), and a pattern speed of $\Omega_{b}=60-70 \mathrm{~km} \mathrm{~s}^{-1} \mathrm{kpc}^{-1}$. A very similar result $\left(\theta=25^{\circ}\right.$ and $\left.\Omega_{b}=50 \mathrm{~km} \mathrm{~s}^{-1} \mathrm{kpc}^{-1}\right)$ was found by Fux $(1997,1999)$ by matching a self-consistent 3D N-body model with stars and gas to the COBE DIRBE data (for stars) and the HI and $\mathrm{CO} l-V$ diagrams (for the gas).

In the last $\sim 10 \mathrm{yrs}$, most of the observational evidence for the presence of a bar in the inner Milky Way has been gathered by studying red clump (RC) stars. The following section is dedicated to these kind of studies. To be complete, however, there are also other recent studies confirming the existence of the main bar, using data from microlensing surveys (e.g., Alcock et al. 2000), or OH/IR and $\mathrm{SiO}$ maser kinematics (Habing et al. 2006).

\subsection{The bar from star counts}

The most robust observational evidence for the presence of a bar was found by means of RC stars used as standard candles, in order to de-project the stellar distribution in the inner Galaxy. The first such work was published by Stanek et al. (1994), who analysed color magnitude diagrams (CMD) from the Optical Gravitational Lensing Experiment (OGLE; Udalski et al. 1992) in three fields, one centred in Baade's Window at $(l, b)=(1,-3.9)$, along the projected minor axis, and the other two at $(l, b)=(-5,-3.5)$ and $(+5,-3.5)$, respectively. The result was that the mean magnitude of the $\mathrm{RC}$ at positive longitudes is $\Delta V_{\mathrm{RC}} \sim 0.2 \mathrm{mag}$ brighter than that in Baade's Window, which in turn is $\Delta V_{\mathrm{RC}} \sim 0.2$ brighter than that at negative longitudes. By assuming that mean age and metallicity of the bulge stellar population does not have a smooth gradient in longitude, this result was interpreted as a smooth variation of the mean distance to the bulge, with longitude, e.g., the presence of a bar. Stanek et al. (1994) measurements were consistent with a bar pivot angle $\theta=45^{\circ}$.

These early results were confirmed by Stanek et al. (1997), Lopez-Corredoira et al. (1997), López-Corredoira et al. (2000), Nikolaev \& Weinberg (1997), Unavane \& Gilmore (1998), Bissantz \& Gerhard (2002), Babusiaux \& Gilmore 
(2005), Benjamin et al. (2005), and Nishiyama et al. (2005). All these studies used RC star counts to constrain some triaxial bar model. The Besançon group (e.g., Picaud \& Robin 2004; Robin et al. 2012) have coupled star counts across the whole CMD with a population synthesis models. They also fit a density model deriving the bar pivot angle and scale lengths. The latter parameters are somewhat different in all the different studies quoted above, with the pivot angle in particular ranging from $10^{\circ}$ to $30^{\circ}$, with occasional values close to $40^{\circ}$, the latter most likely influenced by the presence of the so-called long bar (see Section 5).

A special mention in this context deserves the work by Rattenbury et al. (2007) who fitted a triaxial bar density model to the observed $\mathrm{RC}$ distribution in 44 fields from the OGLE-II survey. With an area coverage about one order of magnitude larger than previous studies $\left(\sim 11 \mathrm{deg}^{2}\right)$, their best fitting pivot angle is $24^{\circ}-27^{\circ}$, the semimajor and semiminor axis scalelengths in the plane ( $x_{0}$ and $y_{0}$, respectively) and vertical scalelengths $\left(z_{0}\right)$ are $\left(x_{0}: y_{0}: z_{0}\right)=(1.2: 0.4: 0.3)$ kpc corresponding to axis ratios $(10: 3.5: 2.6)$. This results have been partially confirmed by Cao et al. (2013) using the final data release of OGLE-III (Soszyński et al. 2011), which covers a much larger area $\left(\sim 90 \mathrm{deg}^{2}\right)$. The triaxial model that best fits the RC distribution is found with adopting a slightly larger pivot angle $\left(29^{\circ}-32^{\circ}\right)$ and axis scalelength $\left(x_{0}: y_{0}: z_{0}\right)=(1.00: 0.41: 0.38) \mathrm{kpc}$.

More recently, the near-IR VISTA Variables in the Vía Láctea ESO Public Survey (Minniti et al. 2010) allowed to map the whole bulge area within $|l|<10^{\circ}$ and $-10<$ $b<+5$ with unprecedented accuracy. The first data release (Saito et al. 2012) allowed Wegg \& Gerhard (2013) to map RC stars across the inner $2.2 \times 1.4 \times 1.1 \mathrm{kpc}$ of the Galactic bar. Their best fitting model has pivot angle of $27^{\circ} \pm 2^{\circ}$, axis ratios $(10: 6.3: 2.6)$ and exponential scalelengths $(0.70: 0.44$ : 0.18) kpc. In Figure 1, we reproduce Figure 17 from Wegg \& Gerhard (2013), showing the bar as viewed from above the plane (note that the $x, y$ axes in this figure do not coincide with the standard X,Y cartesian Galactic coordinates).

\section{THE X-SHAPE}

The presence of a double RC, i.e., with a split in magnitude, in some fields along the projected minor axis $\left(l=0^{\circ}\right)$, in the outer bulge $\left(|b|>5^{\circ}\right)$, was first noticed by McWilliam, Fulbright, \& Rich (2010) and Zoccali (2010) in several different datasets. This result was confirmed by Nataf et al. (2010) using OGLE-II photometry, and by McWilliam \& Zoccali (2010) who interpreted this feature, and the trend of the RC magnitude in the outer bulge as evidence for the outer bulge being X-shaped. This was not seen in previous studies because they were all confined to lower latitudes. One year later, the RC magnitude and density was mapped across the whole bulge area by Saito et al. (2011), using 2MASS photometry (Skrutskie et al. 2006). They confirmed the X-shaped structure seen in the outer bulge, and also demonstrated that this feature was only the outer extension of the main bar. In

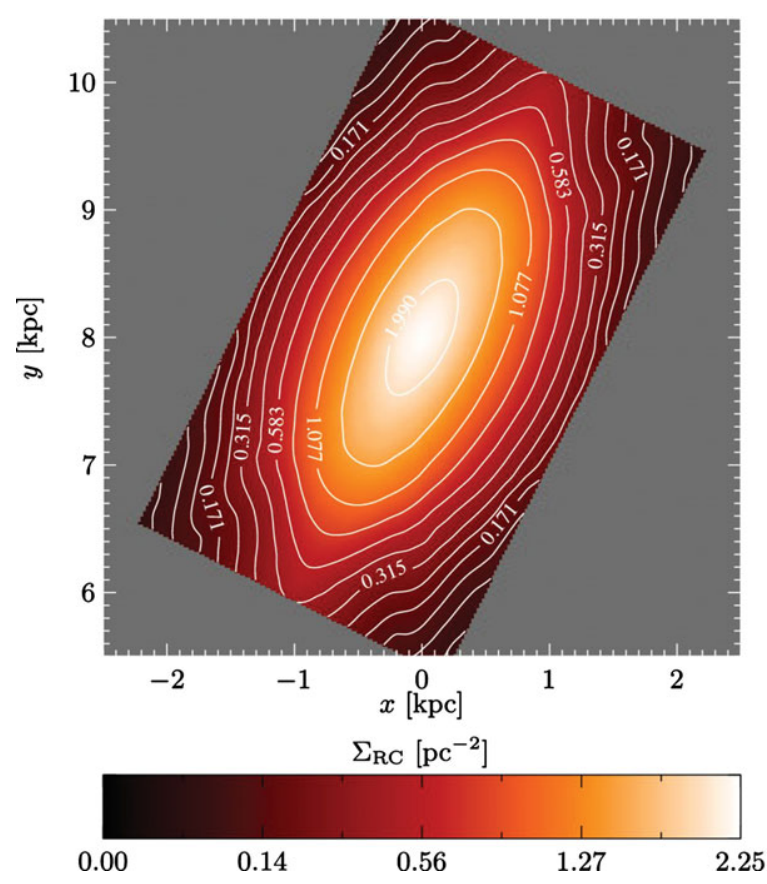

Figure 1. The Galactic bar as seen from the North Galactic Pole. Numbers give the surface density of RC stars in $\mathrm{pc}^{-2}$, contours define isophotes separated by $1 / 3$ mag. Figure reproduced from Wegg \& Gerhard (2013, Mapping the three-dimensional density of the Galactic bulge with VVV red clump stars, their Figure 17).

other words, the two edges of the Galactic bar flare up in two lobes, forming a pronounced peanut, as seen in many external galaxies.

In fact, the boxy morphology is characteristic of all barred galaxies seen edge on (Laurikainen et al. 2014). The peanut shaped (or X-shaped) structure is also a natural product of bar evolution, because dynamical instabilities produce bending and buckling of the elongated stellar orbits within the bars, resulting in the shape of a peanut, or an X-shape when it is more pronounced, when seen edge-on (Patsis, Skokos, \& Athanassoula 2002; Athanassoula 2005a).

Due to a brigther limit magnitude, the 2MASS photometry did not allow to map the regions closer to the Galactic plane, and RC star counts were highly incomplete already at $b \sim 3^{\circ}$. This also prevented a proper normalisation between the inner and outer density, affecting the assessment of the X-shape relevance with respect to the main bar. This problem was overcome thanks to the VVV survey. Indeed the study by Wegg \& Gerhard (2013) mentioned above also included a proper map of the outer, X-shaped bulge. This is shown in Figure 2, reproduced from that paper, where their best fitting bar model is shown edge on, and the shape of the outer isophotes clearly shows the $\mathrm{X}$-shape, or peanut shape.

An alternative explanation of the double $\mathrm{RC}$ has been proposed by Lee, Joo, \& Chung (2015), who claimed that the density and magnitude variation of the RC across the bulge area could also be explained by the presence of a spheroid with two populations of stars, one of which is helium 


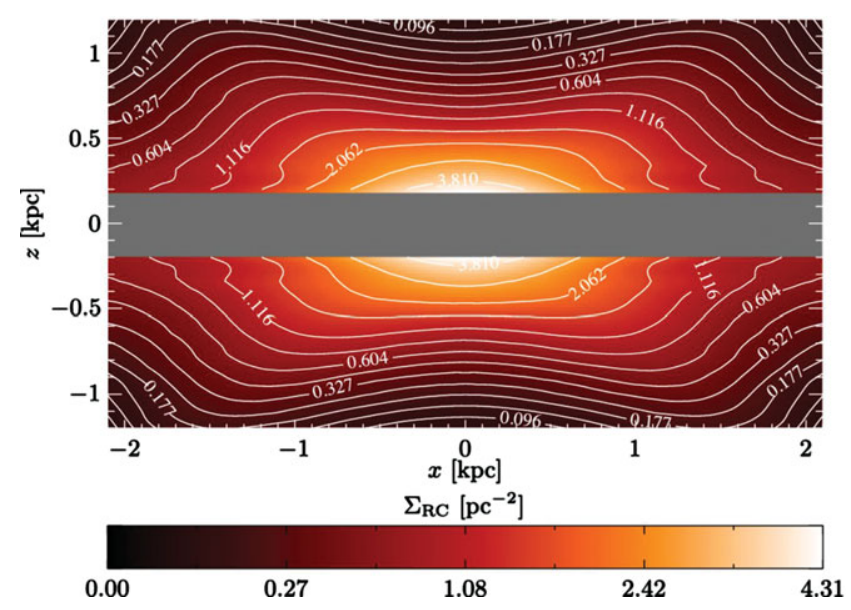

Figure 2. The three-dimensional density of the Milky Way bulge measured in this work projected along the intermediate axis. Numbers give the surface density of RC stars in $\mathrm{pc}^{-2}$, contours define isophotes separated by $1 / 3$ mag. The extinction within $150 \mathrm{pc}$ from the Galactic plane is too high for reliable density measurements, and is therefore excluded from the projection. Figure reproduced from Wegg \& Gerhard (2013, Mapping the three-dimensional density of the Galactic bulge with VVV red clump stars; their Figure 18).

enhanced, similar to Terzan 5 (Ferraro et al. 2009; Massari et al. 2014). This spheroid would therefore show a RC split in magnitude. However, this would only be visible in the outer bulge, because in the inner bulge, it would be erased by the presence of a bar, with a single RC population. The main limitation of this scenario is that it cannot explain the absence of the double RC at $|l|>2^{\circ}$, i.e., away from the projected minor axis, in the outer bulge, as discussed in a rebuttal paper by Gonzalez et al. (2015).

By analysing the correlation between kinematics and metallicity for $\mathrm{K}$ giants in three fields along the bulge projected minor axis, Babusiaux et al. (2010) first suggested that the kinematics of the metal-poor stars $([\mathrm{Fe} / \mathrm{H}] \lesssim 0)$ was consistent with those of a spheroid, while metal-rich stars $([\mathrm{Fe} / \mathrm{H}] \gtrsim 0)$ would have elongated motions typical of galactic bars. More specifically, metal-rich stars have a non-negligible vertex deviation $\left(l_{v} \sim-40\right)$ in Baade's Window, while the metal-poor stars have $l_{v} \sim 0$. In addition, the radial velocity dispersion of the metal-poor stars would stay roughly constant with distance from the plane, while the dispersion of metal-rich stars would be significantly higher closer to the plane. They thus suggested the presence of two different populations in the direction of the Galactic bulge. This suggestion was reinforced by Hill et al. (2011) who show how the metal-rich and metal-poor components have different $[\mathrm{Mg} / \mathrm{Fe}]$ distribution.

An independent confirmation came from Ness et al. (2013) who derived metallicity distribution function of $\sim 14000$ bulge giants in 28 fields across $|l|<30$ and $5<|b|<15$, within the ARGOS survey (Freeman et al. 2013). Thanks to the relatively large magnitude range of their targets, across the RC, they could demonstrate that close to the minor axis, only metal-rich stars would show the split RC indicative of the X-shape. Metal-poor stars, on the contrary, would have a single RC. This demonstrates that metal-poor bulge stars not only have different kinematics but also a different spatial distribution. This result was confirmed by Rojas-Arriagada et al. (2014), using spectroscopic data from the Gaia ESO Survey (Gilmore et al. 2012; Randich, Gilmore, \& Gaia-ESO Consortium 2013).

Dékány et al. (2013) used RRL from OGLE-III and VVV to trace the oldest (and comparatively metal poor) component of the Galactic bulge, and also found that they are arranged in a spheroid with no trace of a bar, nor obviously an X-shape. Same thing was found by Catchpole et al. (2016) using Mira variables. Pietrukowicz et al. (2015) on the contrary, using RRL from the OGLE-IV survey (Soszyński et al. 2014) do confirm the presence of a bar in their spatial distribution, although the extension, ellipticity, and pivot angle of the structure traced by RRL are all significantly smaller than that traced by RC stars.

\section{THE INNER BAR}

About one-third of barred galaxies contain secondary inner bars (Laine et al. 2002; Erwin 2011), whose properties, such as orientation, barycentre and pattern speed affect the gas dynamics of galaxies (Rodriguez-Fernandez \& Combes 2008). Hence, a clear knowledge of the morphology of the innermost bulge regions, and in particular the possible presence of a secondary smaller bar is relevant to our understanding of the Galactic formation and evolution.

The question whether the Milky Way is a double-barred galaxy is still debated. In what follows, we briefly review some of the studies that over the past decade addressed the issue about the existence of an inner bar in the Milky Way. However, it should be noted that this possible inner bar is a structure whose size $(\sim 1 \mathrm{kpc})$ is much larger than the so-called nuclear disk kinematically defined by Schönrich, Aumer, \& Sale (2015).

The presence in the Galactic central region $(R \sim 300 \mathrm{pc})$ of an inner bar nested inside the main bar was first explicitly suggested by Alard (2001). The subtraction of an exponential profile, associated with the main bar, from the deprojected stellar density map, obtained through 2MASS star counts, showed large residuals in the region $|l| \leq 2^{\circ}$ and $|b| \leq 2^{\circ}$. The longitudinal elongation and asymmetry of the derived residuals in the innermost region could be reproduced by a small bar, with steeply dropping density near its edge. Therefore, after ruling out an inappropriate extinction correction and/or a substantial deviation of the main bar density profile from an exponential distribution, as possible cause of the asymmetric residuals, the author concluded that in addition to the main bar, an inner and smaller bar with different orientation might exist.

Later on, Rodriguez-Fernandez \& Combes (2008) used the Alard's stellar density map to constraint the stellar mass distribution adopted in their gas flow dynamical model, which included three components: disk, bulge, and a nuclear bar 
corotating with the main bar (i.e., both bars rotate with the same speed). Their simulation reproduces the longitudevelocity diagram of the Central Molecular Zone as the effect of the nuclear bar on the gas, excluding a possible lopsidedness of the stellar potential due to the nuclear bar. The best-fit model is found for a nuclear bar of mass $(2-5.5) \times 10^{9} \mathrm{M}_{\odot}$, oriented by an angle of $\sim 60^{\circ}-75^{\circ}$ with respect to the SunGalactic Centre line.

However, the question whether the density profile of the central Milky Way region is axisymmetric and its implication for the possible presence of innermost structure date back to earlier time, although admittedly controversial. Indeed, small non-axisymmetric structures were found by Unavane \& Gilmore (1998) as a result of the comparison between star counts in $L$-band observed in two different fields along the Galactic plane at $l= \pm 2.3^{\circ}$. In contrast with this result, van Loon et al. (2003) found that the dereddened luminosity function of point sources at $7 \mu \mathrm{m}$ in the region at $R \leq 1 \mathrm{kpc}$ are extremely symmetric around the bulge minor axis, thus suggesting an azimuthally symmetric spatial distribution of the stellar population.

By using dereddened colour-magnitude diagrams in the $\left(K_{s}\right.$ vs. $H-K_{s}$ ) plane to infer the mean magnitude of the RC peak in the region $b=-1^{\circ}$ and $|l| \leq 10.5^{\circ}$, Nishiyama et al. (2005) observed a clear change in the slope of the RC peak longitudinal distribution. The observed overall variation of the RC peak mean magnitude was consistent with the presence of a main bar whose nearest edge is oriented at positive Galactic latitude (see Section 2.2); however, the shallower profile in the central region $|l| \leq 4^{\circ}$ was interpreted as signature of a distinct inner structure with different orientation angle. The change in orientation of the bar in the central region, as traced by the RC population, was later confirmed by Gonzalez et al. (2011). By using VVV photometry ${ }^{1}$, the authors extended the study of Nishiyama et al. (2005) at latitude $b=+1^{\circ}$ finding an excellent agreement. Figure 3 reproduces the result of Gonzalez et al. (2011, see their Figure 3) by using new and more accurate VVV catalogues obtained with PSF-fitting photometry (see Valenti et al. 2015, for further details). The figure shows a clear change in the RC profile distribution in the innermost region, $R \leq 500 \mathrm{pc}$.

Being detected by independent teams using different dataset and extinction correction, the change of the observed $\mathrm{RC}$ profile slope along the Galactic plane in the region $|b| \leq 1^{\circ}$ is currently widely accepted. Its interpretation as signature of an inner bar is, however, still debated. In this contest, the N-body model of a boxy bulge and bar (with no inner bar) presented by Gerhard \& Martinez-Valpuesta (2012) nicely reproduce the RC profile observed by Gonzalez et al. (2011). The observed variation in the RC slope would then be the result of a change in the stellar density distribution along the line of sight, i.e., from highly elongated to nearly axisymmetric isodensity contours in the innermost regions.

\footnotetext{
${ }^{1}$ DR1 obtained through aperture photometry.
}

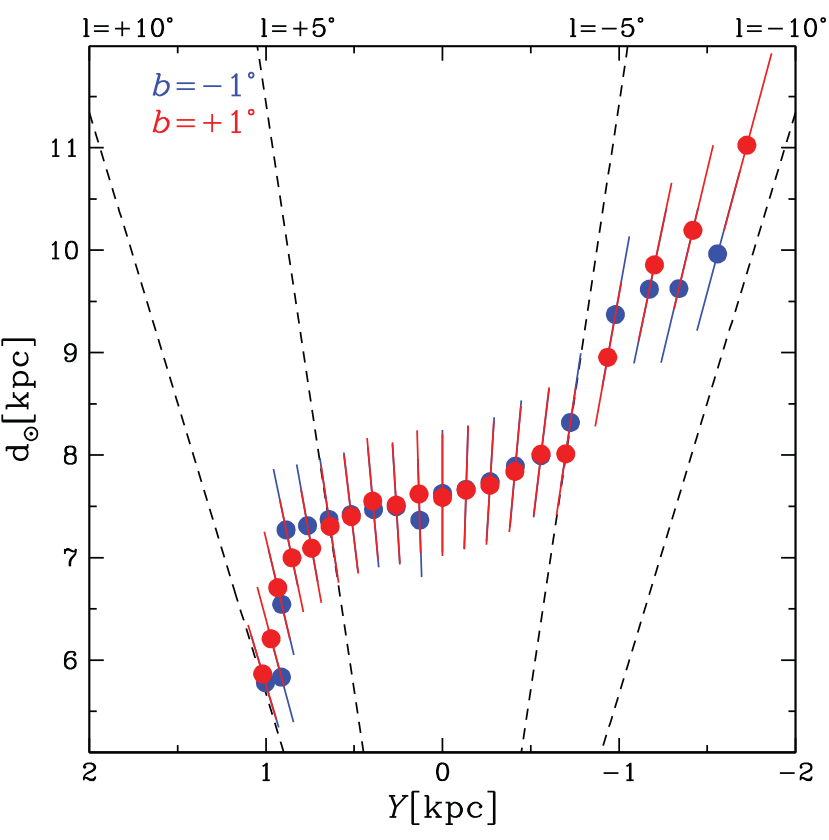

Figure 3. Position of the Galactic bar with respect to the Sun traced by the RC stars, and assuming an absolute magnitude $M_{K}=-1.55$ (Salaris \& Girardi 2002). Blue and red circles respectively show the results for VVV data at latitudes $b=-1^{\circ}$ and $b=+1^{\circ}$, as derived by using the PSF-fitting photometric catalogs fromValenti et al. (2015). As in Figure 3 of Gonzalez et al. (2011), the solid lines identify the distance spread along each line of sight correcting for an intrinsic bulge dispersion of $0.17 \mathrm{mag}$ and photometric errors. Dashed lines refer to the line of sight for longitudes $l= \pm 5^{\circ}, \pm 10^{\circ}$.

Very recent observational evidences seem to support this thesis. In fact, as clearly evident from the VVV-based RC stellar density profile from Valenti et al. (2015), shown here in Figure 4, there is a high-density peak in the innermost region (i.e. $|l| \leq 1^{\circ},|b| \leq 1^{\circ}$ ). In the observed map, the central density contours are slightly asymmetric towards negative longitude. However, when the projection effects along the lines-of-sight are taken into account (see Figure 5) the isodensity contours become progressively less elongated when moving closer to the Galactic centre (compare for instance the shape of the isodensity contours at $b= \pm 4^{\circ}$ and $b= \pm 1^{\circ}$ ). In addition, a tight correlation between the observed VVV density map and the velocity dispersion profile based on the GIBS (Zoccali et al. 2014) kinematics survey has been found, such that the $\sigma$-peak matches the position of the high-density peak (see Figure 3 of Valenti et al. 2015). This seems to confirm that the flattening of the RC mean magnitude profile in the region $|l| \leq 4^{\circ}$ is more likely the result of an inner compact and axisymmetric spheroid rather than the presence of an inner bar.

\section{THE LONG BAR}

Similar to the boxy/peanut structures, the formation of a long bar (i.e., planar bar continuation) is also a common outcome of bar secular evolution (Athanassoula 2005b). As is the case for the main bar, a detailed characterisation of the long 


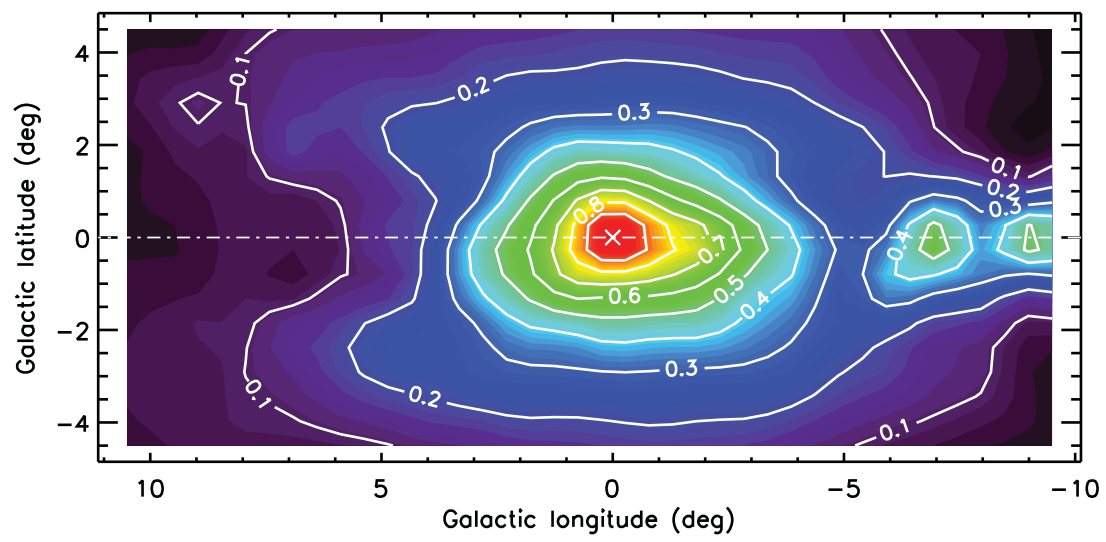

Figure 4. Density map in the longitude-latitude plane based on RC star counts from Valenti et al. (2015). Star counts have been normalised to the Maximum (Max). Solid contours are isodensity curves, linearly spaced by $0.1 \times \operatorname{Max} \mathrm{deg}^{-2}$.

bar (i.e., length, orientation, pattern speed) provides crucial insight to unveil the formation history of our Galaxy. Indeed, numerical modelling have shown that the bar can affect the local stellar velocity distribution of the solar neighbourhood (Dehnen 2000; Minchev et al. 2010), it can be responsible for stellar migration and mixing (Minchev \& Famaey 2010) in the disk, and for the observed non-circular gas flow inside the solar circle (Bissantz, Englmaier, \& Gerhard 2003).

The presence of strong peaks along the Galactic plane at $l \sim 33^{\circ}, 21^{\circ}, 27^{\circ}$, and $-22^{\circ}$ in the near-IR large-scale surface brightness maps, available since the late 70's, stimulated a number of studies aimed at understanding their origin (e.g., Hayakawa et al. 1981; Okuda 1981; Melnick et al. 1987). This, together with the growing perception that the Milky Way was a barred galaxy (see Section 2.1), led to the very first study in which the existence of a long bar was suggested. Combining the COBE/DIRBE surface brightness map with RC star counts from TMGS ${ }^{2} K$-band photometry, Hammersley et al. (1994) demonstrated that the most likely explanation for the presence of the peaks at $15^{\circ} \leq l \leq 35^{\circ}$ in the Galactic plane is that they are associated with star-forming region at the near end of a bar. Their best-fit model is found for a bar of semimajor axis $R=3.7-4 \mathrm{kpc}$ and orientation angle $\theta=75^{\circ}$. Although admittedly the Hammersley et al. (1994) paper never explicitly mentioned the term long bar, the structure suggested and modelled in that work will later on be universally termed as the long bar.

Later on, Hammersley et al. (2000) traced the long bar by looking at the old stellar population selected from nearIR colour-magnitude diagram obtained in regions along the Galactic plane at $l=5^{\circ}, 10^{\circ}, 15^{\circ}, 20^{\circ}, 27^{\circ}, 32^{\circ}$. The differential star counts were found to be fairly similar across the fields at $27^{\circ} \leq l \leq 15^{\circ}$, however, the distance from the Sun of the peak of stars smoothly increased with decreasing lon-

\footnotetext{
${ }^{2}$ Two-Micron Galactic Survey (Garzon et al. 1993).
}

gitude. On the other hand, in the innermost observed fields at $l=5^{\circ}$ and $10^{\circ}$, where the bulge contribution becomes important, the star counts increased considerably. The authors concluded that the peak at $l=27^{\circ}$ could not belong to the bulge, but rather to a different structure such as a long bar, that runs into the bulge. Taking into account the clustering of very young stars at $l=27^{\circ}$ and $21^{\circ}$ found by Hammersley et al. (1994), they suggested that the only component that can reasonably explain all the observational evidences is a bar with half-length $R \sim 4 \mathrm{kpc}$ and a pivot angle $\theta \sim 43^{\circ}$. These results have been substantially confirmed by López-Corredoira et al. (2001) based on DENIS and TMGS star counts map in two off-plane regions at $|b| \approx 1.5^{\circ}$ and $|l| \geq 30^{\circ}$. In addition to the bar position angle $\left(40^{\circ}\right)$ and the semi-major axis ( $3.9 \mathrm{kpc}$ ), they provided a more detailed description of its properties. The closest edge of the bar is found in the first quadrant at $l=27^{\circ}$ at a distance of $5.7 \mathrm{kpc}$ from the Sun (assuming a distance to the Galactic centre $R_{0}=7.9 \mathrm{kpc}$ ), whereas the far end is in the third quadrant at a distance of $11.1 \mathrm{kpc}$ and is seen as the spur extending from the bulge to $l=-14^{\circ}$. The bar scale height, as traced by the young stellar population, is about $50 \mathrm{pc}$, although the old component traced by Hammersley et al. (2000) might have larger scale.

The further advent of accurate and deeper surveys in the near and mid-IR such 2MASS, GLIMPSE, UKIDSS, and VVV has literally promoted a burst in the study of the Galactic long bar, allowing detailed investigations on the presence of star counts asymmetry over homogeneously sampled larger scales. Based on different surveys, independent teams found very similar results constraining to very narrow ranges the bar angle $\left(43^{\circ}-45^{\circ}\right)$ and half-length $(3.7-4.4 \mathrm{kpc})$ (see Benjamin et al. 2005; López-Corredoira et al. 2007; CabreraLavers et al. 2007, 2008; Vallenari, Ragaini, \& Bertelli 2008; Churchwell et al. 2009; Amôres et al. 2013). Moreover, the vast majority of these studies favour the thesis for which the Milky Way hosts two bars: the main bar confined in the bulge within $|l| \leq 10^{\circ}$ with typical angle $\theta \sim 25^{\circ}-30^{\circ}$ (see Section 2.2), and the long bar tilted by $\sim 45^{\circ}$ with respect 

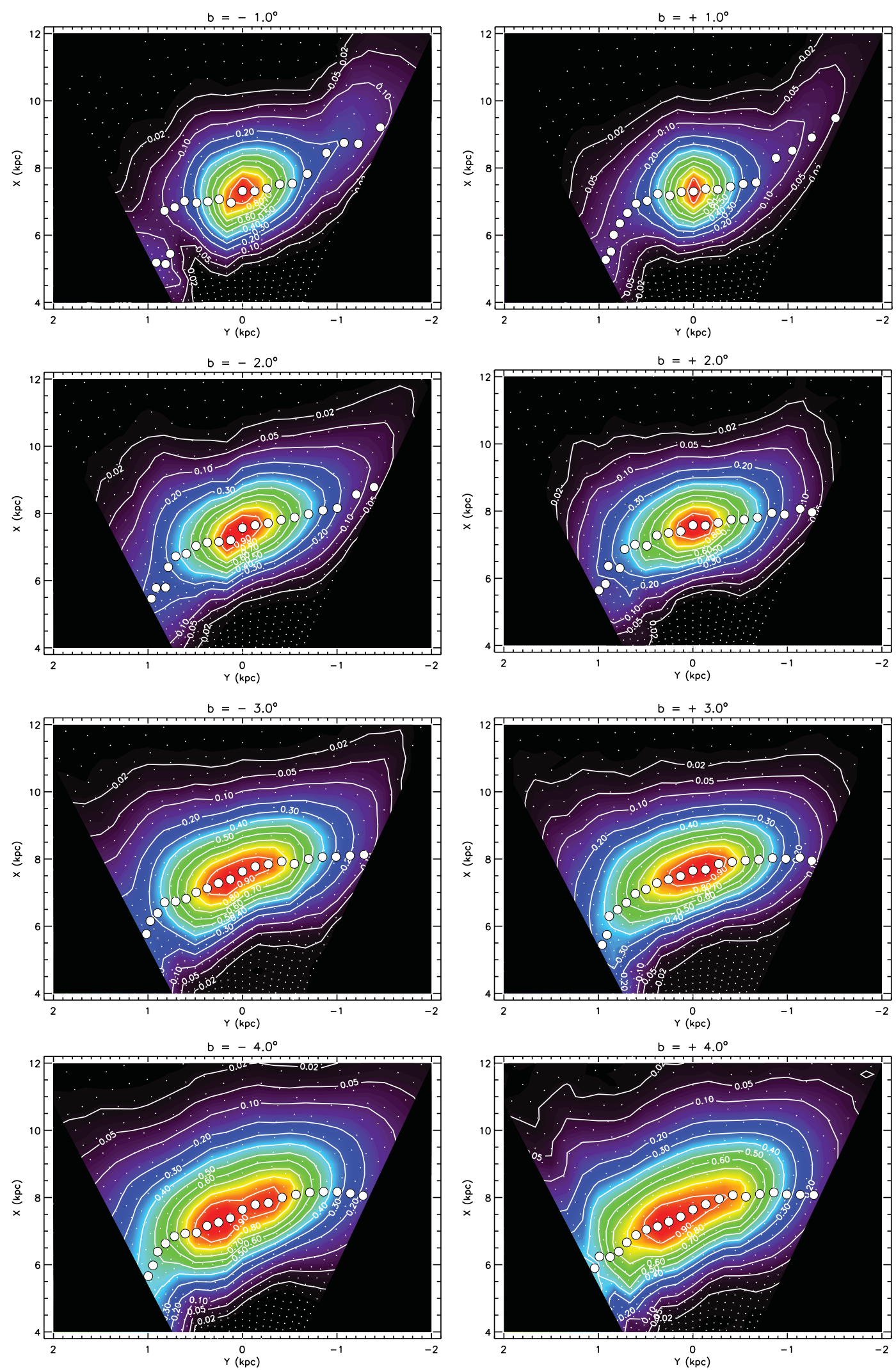

Figure 5. Deprojection of the density map shown in Figure 4 at different latitude. Filled big circles identify the position of the RC peak, whereas small dotted lines refer to the directions $1=1^{\circ}, \pm 2^{\circ}, \pm 3^{\circ}, \pm 4^{\circ}, \pm 5^{\circ}, \pm 6^{\circ}, \pm 7^{\circ}, \pm 8^{\circ}, \pm 9^{\circ}, \pm 10^{\circ}$, as seen from an observer position. 
to the Sun-Galactic Centre line. This configuration of two in-plane misaligned bars has been also detected in three (out of six) external galaxies by Compère, López-Corredoira, \& Garzón (2014), after performing a 3D decomposition modelling, on $K$-band 2MASS images, including three different components: a disk, a bar, and a triaxial bulge.

However, this picture has been very recently challenged by Wegg, Gerhard, \& Portail (2015) who provided a global view of the Milky Way bulge and long bar by using RC stars as distance and density tracers. Combining UKIDSS, VVV, 2MASS, and GLIMPSE data, they obtained a density map of the central $|l|<40^{\circ}$ and $|b|<9^{\circ}$, which is best fitted by a model requiring an orientation angle of the long bar consistent with that of the triaxial bulge (i.e., $28^{\circ}-33^{\circ}$ ). The scale height as traced by the RC stars changes smoothly from the bulge to the long bar. They found evidence for two scales height in the long bar, suggesting the presence of a $\sim 180 \mathrm{pc}$ thin bar component whose density decreases outwards, and a $\sim 45 \mathrm{pc}$ superthin component whose half-length is $\sim 4.6 \mathrm{kpc}$. According to the authors, the thin bar could be the barred counterpart of the solar neighbourhood thin disc, whereas the superthin one could be associated to younger stars $(\sim 1 \mathrm{Gyr})$ towards the end of the bar.

In agreement with N-body simulations (MartinezValpuesta \& Gerhard 2011; Romero-Gómez et al. 2011), their findings support the scenario in which the long and main bar are two parts of the same structure, and that the Milky Way contains a central boxy/peanut bulge which is the vertical extension of a longer, flatter bar.

On the other hand, the presence of two in-plane bars, with the long component twisted with respect to the barred bulge is hardly predicted by dynamical models because two separate rotating bars should align with each other through dynamical coupling in less than a few rotation periods. In this framework, Martinez-Valpuesta \& Gerhard (2011) showed that by using a dynamical model of a single stellar bar evolved from the disk, and a boxy bulge originated from it, through secular evolution and buckling instability, can reproduce the observations. In particular, the observed mismatch between the orientation of the long $\left(45^{\circ}\right)$ and main $\left(27^{\circ}\right)$ bar is caused by a combination of volume effect and variation of the density distribution along the observer line of sight. However, regardless from the problem of the current $\mathrm{N}$-body dynamical simulations to reproduce the possible two bar configurations, the puzzling fact remains that, although using the same tracers (i.e., RC stars), methodology, and dataset (i.e., UKIDSS) Cabrera-Lavers et al. (2008) and Wegg et al. (2015) derived very different results.

\section{SUMMARY AND DISCUSSION}

In this review, we provided a description of the 3D structure of the Milky Way bulge from observational perspectives.

Over the years, since the pioneering work of de Vaucouleurs (1964), there has been a large number of studies addressing the problem of the existence of the bar, and aimed at characterising its main properties. By using a variety of techniques, ranging from the integrated photometry and star counts to gas kinematics and microlensing, these works contributed to build and strengthen the general consensus that our bulge is indeed a bar. The near side of the bar is in the first Galactic quadrant, and its orientation with respect to the Sun-Galactic Centre line-of-sight is $\sim 27^{\circ}$.

The advent of IR surveys (e.g., COBE/DIRBE, 2MASS, and VVV), allowing to uniformly map large scale area (e.g. $\geq 300 \mathrm{deg}^{2}$ ), has finally provided a comprehensive view of the Milky Way bulge as a whole revealing its boxy/peanut structure. This morphology is typical of bulges formed out of the natural evolution of edge-on barred galaxies, as consequence of disk instabilities and bar vertical buckling. Moreover, the observed magnitude split of the RC in the outer region of the bulge, is interpreted as evidence of an X-shaped structure, i.e., a pronounced boxy/peanut shape, which the models explain as the result of bar growing.

The extensive studies of the innermost region of the bulge (i.e., $|l| \leq 2$ and $|b| \leq 2$ ) unveiled the presence of high axisymmetric stellar density peak. The latter, rather than the presence of an inner bar, seems to be responsible for the change in the bar pivot angle in this region. Moreover, the central density peak matches the peak found in the stellar radial velocity dispersion.

Several studies focussing on the region along the Galactic plane, and outside the main bar, disclosed the existence of a long bar, with semimajor axis of $\sim 4.7 \mathrm{kpc}$ in length, and misaligned with respect to the bulge main bar. However, the scenario of a triaxial bulge with two in-plane bars with different orientation has been challenged by a very recent new results that, instead, suggests that the long bar is just the extension at higher longitude of the bulge main bar.

The correlation between metallicity and kinematics of bulge giants, as well as the distribution of RRL variables suggest the presence in the Milky Way bulge of a metalpoor spheroid. However, while the general properties in term of morphology, kinematics, and chemical content of the boxy/peanut structure have been extensively studied, the characterisation of the metal-poor spheroids is far for being complete. Additional detailed investigation aimed at confirming whether or not giants and RRL variables trace different structure, and characterising the kinematics of the variables population, is still needed.

\section{ACKNOWLEDGEMENTS}

We thank Chris Wegg for providing Figures 1 and 2, and the editors for inviting us to write this review. MZ gratefully acknowledge support by the Ministry of Economy, Development, and Tourism's Millennium Science Initiative through grant IC120009, awarded to The Millennium Institute of Astrophysics (MAS), by Fondecyt Regular 1150345, by the BASAL-CATA Center for Astrophysics and Associated Technologies PFB-06 and by CONICYT's PCI programme through grant DPI20140066. 


\section{REFERENCES}

Alard, C. 2001, A\&A, 379, L44

Alcock, C., et al. 2000, ApJ, 541, 734

Amôres, E. B., López-Corredoira, M., González-Fernández, C., Moitinho, A., Minniti, D., \& Gurovich, S. 2013, A\&A, 559, A11

Athanassoula, E. 2005a, MNRAS, 358, 1477

Athanassoula, E. 2005b, MNRAS, 358, 1477

Babusiaux, C., \& Gilmore, G. 2005, MNRAS10.1111/j.13652966.2005.08828.x, 358, 1309

Babusiaux, C., et al. 2010, A\&A, 519, A77

Bell, E. F., et al. 2008, ApJ, 680, 295

Benjamin, R. A., et al. 2005, ApJ, 630, L149

Bensby, T., et al. 2011, A\&A, 533, A134

Bensby, T., et al. 2013, A\&A, 549, A147

Binney, J., Gerhard, O., \& Spergel, D. 1997, MNRAS, 288, 365

Bissantz, N., \& Gerhard, O. 2002, MNRAS, 330, 591

Bissantz, N., Englmaier, P., \& Gerhard, O. 2003, MNRAS, 340, 949

Blitz, L., \& Spergel, D. N. 1991, ApJ, 379, 631

Bournaud, F., Elmegreen, B. G., \& Martig, M. 2009, ApJ, 707, L1

Cabrera-Lavers, A., González-Fernández, C., Garzón, F., Hammersley, P. L., \& López-Corredoira, M. 2008, A\&A, 491, 781

Cabrera-Lavers, A., Hammersley, P. L., González-Fernández, C., López-Corredoira, M., Garzón, F., \& Mahoney, T. J. 2007, A\&A, 465,825

Calamida, A., et al. 2015, ApJ, 810, 8

Cao, L., Mao, S., Nataf, D., Rattenbury, N. J., \& Gould, A. 2013 , MNRAS, 434, 595

Carollo, C. M., Scarlata, C., Stiavelli, M., Wyse, R. F. G., \& Mayer, L. 2007, ApJ, 658, 960

Catchpole, R. M., Whitelock, P. A., Feast, M. W., Hughes, S. M. G., Irwin, M., \& Alard, C. 2016, MNRAS, 455, 2216

Churchwell, E., et al. 2009, PASP, 121, 213

Clarkson, W. I., et al. 2011, ApJ, 735, 37

Compère, P., López-Corredoira, M., \& Garzón, F. 2014, A\&A, 571, A98

de Vaucouleurs, G. 1964, in IAU Symp., Vol. 20, The Galaxy and the Magellanic Clouds, ed. K. J. Kerr (Canberra: Australian Academy of Science), 195

Dehnen, W. 2000, AJ, 119, 800

Dékány, I., Minniti, D., Catelan, M., Zoccali, M., Saito R. K., Hempel, M., \& Gonzalez, O. A. 2013, ApJ, 776, L19

Dwek, E., et al. 1995, ApJ, 445, 716

Elmegreen, B. G., Bournaud, F., \& Elmegreen, D. M. 2008, ApJ, 688, 67

Erwin, P. 2011, MSAIS, 18, 145

Ferraro, F. R., et al. 2009, Natur, 462, 1028

Freeman, K., et al. 2013, MNRAS, 428, 3660

Fux, R. 1997, A\&A, 327, 983

Fux, R. 1999, A\&A, 345, 787

Garzon, F., Hammersley, P. L., Mahoney, T., Calbet, X., Selby, M. J., \& Hepburn, I. D. 1993, MNRAS, 264, 773

Gerhard, O., \& Martinez-Valpuesta, I. 2012, ApJ, 744, L8

Gilmore, G., et al. 2012, Msngr, 147, 25

Gonzalez, O. A., Rejkuba, M., Minniti, D., Zoccali, M., Valenti, E., \& Saito, R. K. 2011, A\&A, 534, L14

Gonzalez, O. A., Zoccali, M., Debattista, V. P., Alonso-García J., Valenti, E., \& Minniti, D. 2015, A\&A, 583, L5
Habing, H. J., Sevenster, M. N., Messineo, M., van de Ven, G., \& Kuijken, K. 2006, A\&A, 458, 151

Hammersley, P. L., Garzon, F., Mahoney, T., \& Calbet, X. 1994, MNRAS, 269, 753

Hammersley, P. L., Garzón, F., Mahoney, T. J., López-Corredoira M., \& Torres, M. A. P. 2000, MNRAS, 317, L45

Hauser, M. G., et al.1990, in Astrophysics and Space Science Library, Vol. 166, IAU Colloq. 123, Observatories in Earth Orbit and Beyond, ed. Y. Kondo (Dordrecht: Kluwer), 19

Hayakawa, S., Matsumoto, T., Murakami, H., Uyama, K., Thomas, J. A., \& Yamagami, T. 1981, A\&A, 100, 116

Hill, V., et al. 2011, A\&A, 534, A80

Immeli, A., Samland, M., Gerhard, O., \& Westera, P. 2004, A\&A, 413, 547

Kent, S. M. 1992, ApJ, 387, 181

Kent, S. M., Dame, T. M., \& Fazio, G. 1991, ApJ, 378, 131

Kormendy, J., \& Kennicutt, Jr, R. C. 2004, ARA\&A, 42, 603

Kuijken, K., \& Rich, R. M. 2002, AJ, 124, 2054

Laine, S., Shlosman, I., Knapen, J. H., \& Peletier, R. F. 2002, ApJ, 567, 97

Laurikainen, E., Salo, H., Athanassoula, E., Bosma, A., \& HerreraEndoqui, M. 2014, MNRAS, 444, L80

Lee, Y.-W., Joo, S.-J., \& Chung, C. 2015, MNRAS, 453, 3906

López-Corredoira, M., Cabrera-Lavers, A., Mahoney, T. J., Hammersley, P. L., Garzón, F., \& González-Fernández, C. 2007, AJ, 133, 154

Lopez-Corredoira, M., Garzon, F., Hammersley, P., Mahoney, T., \& Calbet, X. 1997, MNRAS, 292, L15

López-Corredoira, M., Hammersley, P. L., Garzón, F., CabreraLavers, A., Castro-Rodríguez, N., Schultheis, M., \& Mahoney, T. J. 2001, A\&A, 373, 139

López-Corredoira, M., Hammersley, P. L., Garzón, F., Simonneau E., \& Mahoney, T. J. 2000, MNRAS, 313, 392

Martinez-Valpuesta, I., \& Gerhard, O. 2011, ApJ, 734, L20

Massari, D., et al. 2014, ApJ, 795, 22

Matsumoto, T., Hayakawa, S., Koizumi, H., Murakami, H., Uyama, K., Yamagami, T., \& Thomas, J. A. 1982, in AIP Conf. Proc., Vol. 83, The Galactic Center, ed. G. R. Riegler, \& R. D. Blandford (New York: Am. Inst. Phys.), 48,

McWilliam, A., Fulbright, J., \& Rich, R. M. 2010, in IAU Symp., Vol. 265, Chemical Abundances in the Universe: Connecting First Stars to Planets, ed. K. Cunha, M. Spite, \& B. Barbuy (Cambridge: Cambridge Univ. Press), 279,

McWilliam, A., \& Zoccali, M. 2010, ApJ, 724, 1491

Melnick, G. J., Fazio, G. G., Koch, D. G., Rieke, G. H., Young, E. T., Low, F. J., Hoffmann, W. F., \& Gautier, T. N. 1987, in AIP Conf. Proc., Vol. 155, The Galactic Center, ed. D. C. Backer (New York: Am. Inst. Phys.), 157

Minchev, I., Boily, C., Siebert, A., \& Bienayme, O. 2010, MNRAS, 407, 2122

Minchev, I., \& Famaey, B. 2010, ApJ, 722, 112

Minniti, D., et al. 2010, NewA, 15, 433

Nataf, D. M., Udalski, A., Gould, A., Fouqué, P., \& Stanek, K. Z. 2010, ApJ, 721, L28

Ness, M., et al. 2013, MNRAS, 430, 836

Nikolaev, S., \& Weinberg, M. D. 1997, ApJ, 487, 885

Nishiyama, S., et al. 2005, ApJ, 621, L105

Okuda, H. 1981, in IAU Symp., Vol. 96, Infrared Astronomy, ed. C. G. Wynn-Williams, D. P. Cruikshank (Dordrecht: Reidel), 247 
Ortolani, S., Renzini, A., Gilmozzi, R., Marconi, G., Barbuy, B., Bica, E., \& Rich, R. M. 1995, Nature, 377, 701

Patsis, P. A., Skokos, C., \& Athanassoula, E. 2002, MNRAS, 337, 578

Picaud, S., \& Robin, A. C. 2004, A\&A, 428, 891

Pietrukowicz, P., et al. 2015, ApJ, 811, 113

Randich, S., Gilmore, G., \& Gaia-ESO Consortium 2013, Msngr, 154,47

Rattenbury, N. J., Mao, S., Sumi, T., \& Smith, M. C. 2007, MNRAS, 378, 1064

Robin, A. C., Marshall, D. J., Schultheis, M., \& Reylé, C. 2012, A\&A, 538, A106

Robin, A. C., Reylé, C., Derrière, S., \& Picaud, S. 2003, A\&A, 409, 523

Rodriguez-Fernandez, N. J., \& Combes, F. 2008, A\&A, 489, 115

Rojas-Arriagada, A., et al. 2014, A\&A, 569, A103

Romero-Gómez, M., Athanassoula, E., Antoja, T., \& Figueras, F. 2011, MNRAS, 418, 1176

Sahu, K. C., et al. 2006, Nature, 443, 534

Saito, R. K., Zoccali, M., McWilliam, A., Minniti, D., Gonzalez, O. A., \& Hill, V. 2011, AJ, 142, 76

Saito, R. K., et al. 2012, A\&A, 537, A107

Salaris, M., \& Girardi, L. 2002, MNRAS, 337, 332

Schönrich, R., Aumer, M., \& Sale, S. E. 2015, ApJ, 812, L21

Skrutskie, M. F., et al. 2006, AJ, 131, 1163

Soszyński, I., et al. 2011, AcA, 61, 1

Soszyński, I., et al. 2014, AcA, 64, 177
Stanek, K. Z., Mateo, M., Udalski, A., Szymanski, M., Kaluzny, J., \& Kubiak, M. 1994, ApJ, 429, L73

Stanek, K. Z., Udalski, A., SzymaŃski, M., KaŁuŻny, J., Kubiak, Z. M., Mateo, M., \& KrzemiŃski, W. 1997, ApJ, 477, 163

Udalski, A., Szymanski, M., Kaluzny, J., Kubiak, M., \& Mateo, M. 1992, AcA, 42, 253

Unavane, M., \& Gilmore, G. 1998, MNRAS, 295, 145

Valenti, E., Zoccali, M., Renzini, A., Brown, T. M., Gonzalez, O. A., Minniti, D., Debattista, V. P., \& Mayer, L. 2013, A\&A, 559, A98

Valenti, E., et al. 2016, A\&A, 587, L6

Vallenari, A., Ragaini, S., \& Bertelli, G. 2008, in IAU Symp., Vol. 245, Formation and Evolution of Galaxy Bulges, ed. M. Bureau, E. Athanassoula, \& B. Barbuy (Cambridge: Cambridge Univ. Press) 371

van Loon, J. T., et al. 2003, MNRAS, 338, 857

Wegg, C., \& Gerhard, O. 2013, MNRAS, 435, 1874

Wegg, C., Gerhard, O., \& Portail, M. 2015, MNRAS, 450, 4050

Weiland, J. L., et al. 1994, ApJ, 425, L81

Zoccali, M. 2010, in IAU Symp., Vol. 265, Chemical Abundances in the Universe: Connecting First Stars to Planets, ed. K. Cunha, M. Spite, \& B. Barbuy (Cambridge: Cambridge Univ. Press), 271

Zoccali, M., Cassisi, S., Frogel, J. A., Gould, A., Ortolani, S., Renzini, A., Rich, R. M., \& Stephens, A. W. 2000, ApJ, 530, 418

Zoccali, M., et al. 2003, A\&A, 399, 931

Zoccali, M., et al. 2014, A\&A, 562, A66 